\title{
Newton Type Iteration for Tikhonov Regularization of Nonlinear Ill-Posed Problems in Hilbert Scales
}

\author{
Monnanda Erappa Shobha and Santhosh George \\ Department of Mathematical and Computational Sciences, National Institute of Technology Karnataka, Mangalore 575 025, India
}

Correspondence should be addressed to Monnanda Erappa Shobha; shobha.me@gmail.com

Received 13 February 2014; Accepted 11 June 2014; Published 1 July 2014

Academic Editor: Beny Neta

Copyright (C) 2014 M. E. Shobha and S. George. This is an open access article distributed under the Creative Commons Attribution License, which permits unrestricted use, distribution, and reproduction in any medium, provided the original work is properly cited.

Recently, Vasin and George (2013) considered an iterative scheme for approximately solving an ill-posed operator equation $F(x)=y$. In order to improve the error estimate available by Vasin and George (2013), in the present paper we extend the iterative method considered by Vasin and George (2013), in the setting of Hilbert scales. The error estimates obtained under a general source condition on $x_{0}-\widehat{x}$ ( $x_{0}$ is the initial guess and $\widehat{x}$ is the actual solution), using the adaptive scheme proposed by Pereverzev and Schock (2005), are of optimal order. The algorithm is applied to numerical solution of an integral equation in Numerical Example section.

\section{Introduction}

In this study, we are interested in approximately solving a nonlinear ill-posed operator equation:

$$
F(x)=y
$$

where $F: D(F) \subseteq X \rightarrow Y$ is a nonlinear operator. Here $D(F)$ is the domain of $F$, and $\langle\cdot, \cdot\rangle$ is the inner product with corresponding norm $\|\cdot\|$ on the Hilbert spaces $X$ and $Y$. Throughout this paper we denote by $B_{r}\left(x_{0}\right)$ the ball of radius $r$ centered at $x_{0}, F^{\prime}(x)$ denotes the Fréchet derivative of $F$ at $x \in D(F)$, and $F^{\prime *}(\cdot)$ denotes the adjoint of $F^{\prime}(\cdot)$. We assume that $y^{\delta} \in Y$ are the available noisy data satisfying

$$
\left\|y-y^{\delta}\right\| \leq \delta
$$

where $\delta$ is the noise level. Equation (1) is, in general, ill-posed, in the sense that a unique solution that depends continuously on the data does not exist. Since the available data is $y^{\delta}$, one has to solve (approximately) the perturbed equation

$$
F(x)=y^{\delta}
$$

instead of (1).
To solve the ill-posed operator equations, various regularization methods are used, for example, Tikhonov regularization, Landweber iterative regularization, LevenbergMarquardt method, Lavrentiev regularization, Newton type iterative method, and so forth (see, e.g., [1-16]).

In [16], Vasin and George considered the iteration (which is a modified form of the method considered in [8])

$$
x_{n+1, \alpha}^{\delta}:=x_{n, \alpha}-R_{\alpha}^{-1}\left[A_{0}^{*}\left(F\left(x_{n, \alpha}^{\delta}\right)-y^{\delta}\right)+\alpha\left(x_{n, \alpha}^{\delta}-x_{0}\right)\right],
$$

where $R_{0}:=A_{0}^{*} A_{0}+\beta I, A_{0}=F^{\prime}\left(x_{0}\right), x_{0, \alpha}^{\delta}=x_{0}$ is the initial guess, $\alpha>0$ is the regularization parameter, and $\beta>\alpha$. Iteration (4) was used to obtain an approximation for the zero $x_{\alpha}^{\delta}$ of $F^{\prime}\left(x_{0}\right)^{*}\left(F\left(x_{0}\right)-y^{\delta}\right)+\alpha\left(x-x_{0}\right)=0$ and proved that $x_{\alpha}^{\delta}$ is an approximate solution of (1). The regularization parameter $\alpha$ in [16] was chosen appropriately from the finite set $D_{N}:=\left\{\alpha_{i}: 0<\alpha_{0}<\alpha_{1}<\cdots<\alpha_{N}\right\}$ depending on the inexact data $y^{\delta}$ and the error level $\delta$ satisfying (2) using the adaptive parameter selection procedure suggested by Pereverzev and Schock [17].

In order to improve the rate of convergence many authors have considered the Hilbert scale variant of the regularization 
methods for solving ill-posed operator equations, for example, [18-26]. In this study, we present the Hilbert scale variant of (4).

We consider the Hilbert scale $\left\{X_{t}\right\}_{t \in \mathbb{R}}$ (see $[14,18,23,26-$ 29]) generated by a strictly positive self-adjoint operator $B$ : $D(B) \subset X \rightarrow X$, with the domain $D(B)$ dense in $X$ satisfying $\|B x\| \geq\|x\|$, for all $x \in D(B)$. Recall $[19,28]$ that the space $X_{t}$ is the completion of $D:=\bigcap_{k=0}^{\infty} D\left(B^{k}\right)$ with respect to the norm $\|x\|_{t}$, induced by the inner product

$$
\langle u, v\rangle_{t}=\left\langle B^{t / 2} u, B^{t / 2} v\right\rangle, \quad u, v \in D(B) .
$$

In this paper, we consider the sequence $\left\{x_{n, \alpha, s}^{\delta}\right\}$ defined iteratively by

$$
\begin{aligned}
& x_{n+1, \alpha, s}^{\delta} \\
& \quad=x_{n, \alpha, s}^{\delta}-R_{\beta}^{-1}\left[A_{0}^{*}\left(F\left(x_{n, \alpha, s}^{\delta}\right)-y^{\delta}\right)+\alpha B^{s}\left(x_{n, \alpha, s}^{\delta}-x_{0}\right)\right],
\end{aligned}
$$

where $R_{\beta}^{-1}:=\left(A_{0}^{*} A_{0}+\beta B^{s}\right)^{-1}, x_{0, \alpha, s}^{\delta}:=x_{0}$, is the initial guess, $\beta>\alpha$, for obtaining an approximation for zero $x_{\alpha, s}^{\delta}$ of (cf. $[21,30])$

$$
A_{0}^{*}\left(F(x)-y^{\delta}\right)+\alpha B^{s}\left(x-x_{0}\right)=0 .
$$

As in [16], we use the following center-type Lipschitz condition for the convergence of the iterative scheme.

Assumption 1. Let $x_{0} \in X$ be fixed. There exists a constant $k_{0}$ such that, for every $u \in B_{r}\left(x_{0}\right) \cup B_{r}(\widehat{x}) \subseteq D(F)$ and $v \in X$, there exists an element $\Phi\left(x_{0}, u, v\right) \in X$ satisfying

$$
\begin{gathered}
{\left[F^{\prime}\left(x_{0}\right)-F^{\prime}(u)\right] v=F^{\prime}\left(x_{0}\right) \Phi\left(x_{0}, u, v\right),} \\
\left\|\Phi\left(x_{0}, u, v\right)\right\| \leq k_{0}\|v\|\left\|x_{0}-u\right\| .
\end{gathered}
$$

The error estimates in this work are obtained using the source condition on $x_{0}-\widehat{x}$. In addition to the advantages listed in [16, see page 3], the method considered in this paper gives optimal order for a range of values of smoothness assumptions on $x_{0}-\widehat{x}$. The regularization parameter $\alpha$ is chosen from some finite set $\left\{\alpha_{0}<\alpha_{1}<\alpha_{2} \cdots<\alpha_{N}\right\}$ using the balancing principle considered by Pereverzev and Schock in [17].

The paper is organized as follows. In Section 2, we give the analysis of the method for regularization of (6) in the setting of Hilbert scales. The error analysis and adaptive scheme of parameter $\alpha$ are given in Section 3. In Section 4, implementation of the method along with a numerical example is presented to validate the efficiency of the proposed method and we conclude the paper in Section 5.

\section{The Method}

First we will prove that the sequence $\left(x_{n, \alpha, s}^{\delta}\right)$ defined by (6) converges to the zero $x_{\alpha, s}^{\delta}$ of (7) and then we show that $x_{\alpha, s}^{\delta}$ is an approximation to the solution $\widehat{x}$ of (1).
Let $A_{s}=A_{0} B^{-s / 2}$. We make use of the relation

$$
\left\|\left(A_{s}^{*} A_{s}+\alpha I\right)^{-1}\left(A_{s}^{*} A_{s}\right)^{p}\right\| \leq \alpha^{p-1}, \quad p>0,0<p \leq 1,
$$

which follows from the spectral properties of the positive selfadjoint operator $A_{s}^{*} A_{s}, s>0$. Usually, for the analysis of regularization methods in Hilbert scales, an assumption of the form (cf. $[18,24])$

$$
\left\|F^{\prime}(\widehat{x}) x\right\| \sim\|x\|_{-b}, \quad x \in X
$$

on the degree of ill-posedness is used. In this paper instead of (10) we require only a weaker assumption:

$$
d_{1}\|x\|_{-b} \leq\left\|A_{0} x\right\| \leq d_{2}\|x\|_{-b}, \quad x \in D(F),
$$

for some positive reals $b, d_{1}$, and $d_{2}$.

Note that (11) is simpler than that of (10). Now we define $f$ and $g$ by

$$
\begin{array}{r}
f(t)=\min \left\{d_{1}^{t}, d_{2}^{t}\right\}, \quad g(t)=\max \left\{d_{1}^{t}, d_{2}^{t}\right\}, \\
t \in \mathbb{R},|t| \leq 1 .
\end{array}
$$

The following proposition is used for further analysis.

Proposition 2 (cf. see [29, Proposition 2.1]). For $s>0$ and $|\nu| \leq 1$,

$$
f(\nu)\|x\|_{-\nu(s+b)} \leq\left\|\left(A_{s}^{*} A_{s}\right)^{v / 2} x\right\| \leq g(\nu)\|x\|_{-\gamma(s+b)}, \quad x \in H .
$$

Let us define a few parameters essential for the convergence analysis. Let

$$
\begin{gathered}
\psi_{2}(s):=\frac{g(s /(s+b))}{f(s /(s+b))} ; \quad \overline{\psi_{2}(s)}:=\frac{1}{f(s /(s+b))} \\
e_{n, \alpha, s}^{\delta}:=\left\|x_{n, \alpha, s}^{\delta}-x_{n-1, \alpha, s}^{\delta}\right\|, \quad \forall n \geq 0 \\
\delta_{0}<\frac{\beta^{b / 2(s+b)}}{2 k_{0} \overline{\psi_{2}(s)}}\left(\psi_{2}(s)+\frac{\alpha_{0}^{2}}{2 \beta^{2}}\right) \\
\left\|\hat{x}-x_{0}\right\| \leq \rho
\end{gathered}
$$

with

$$
\begin{aligned}
& \rho< \frac{-1}{k_{0}}+\frac{1}{k_{0} \psi_{2}(s)} \\
& \times \sqrt{\psi_{2}(s)\left[\left(\frac{\alpha^{2}}{2 \beta^{2}}+\psi_{2}(s)\right)-2 k_{0} \overline{\psi_{2}(s)} \beta^{-b / 2(s+b)} \delta\right]}, \\
& \gamma_{\rho}:=\overline{\psi_{2}(s)} \beta^{-b / 2(s+b)} \delta+\psi_{2}(s)\left(\frac{k_{0}}{2} \rho^{2}+\rho\right) .
\end{aligned}
$$


Journal of Mathematics

3

Further let $\gamma_{\rho}<\alpha_{0}^{2} / 4 k_{0} \beta^{2}$, and

$$
\begin{gathered}
r_{1}=\frac{\alpha+\sqrt{\alpha^{2}-4 k_{0} \gamma_{\rho} \beta^{2}}}{2 k_{0} \beta}, \\
r_{2}=\min \left\{\frac{1}{\psi_{2}(s) k_{0}}, \frac{\alpha-\sqrt{\alpha^{2}-4 k_{0} \gamma_{\rho} \beta^{2}}}{2 k_{0} \beta}\right\} .
\end{gathered}
$$

For $r \in\left(r_{1}, r_{2}\right)$, let

$$
q=\psi_{2}(s)\left(k_{0} r+\frac{\beta-\alpha}{\beta}\right) .
$$

Then $q<1$.

Lemma 3. Let Proposition 2 hold. Then for all $h \in X$, the following hold:

(a) $\left\|\left(A_{0}^{*} A_{0}+\beta B^{s}\right)^{-1} A_{0}^{*} A_{0} h\right\| \leq \psi_{2}(s)\|h\|$,

(b) $\left\|\left(A_{0}^{*} A_{0}+\beta B^{s}\right)^{-1} B^{s} h\right\| \leq \psi_{2}(s)(1 / \beta)\|h\|$.

Proof. Observe that, by Proposition 2,

$$
\begin{aligned}
& \left\|\left(A_{0}^{*} A_{0}+\beta B^{s}\right)^{-1} A_{0}^{*} A_{0} h\right\| \\
& =\left\|B^{-s / 2}\left(A_{s}^{*} A_{s}+\beta I\right)^{-1} A_{s}^{*} A_{s} B^{s / 2} h\right\| \\
& \leq \frac{1}{f(s /(s+b))} \\
& \times\left\|\left(A_{s}^{*} A_{s}\right)^{s / 2(s+b)}\left(A_{s}^{*} A_{s}+\beta I\right)^{-1}\left(A_{s}^{*} A_{s}\right) B^{s / 2} h\right\| \\
& \leq \frac{1}{f(s /(s+b))}\left\|\left(A_{s}^{*} A_{s}+\beta I\right)^{-1} A_{s}^{*} A_{s}\right\| \\
& \times\left\|\left(A_{s}^{*} A_{s}\right)^{s / 2(s+b)} B^{s / 2} h\right\| \\
& \leq \frac{g(s /(s+b))}{f(s /(s+b))}\left\|B^{s / 2} h\right\|_{-s} \\
& \leq \frac{g(s /(s+b))}{f(s /(s+b))}\|h\|, \\
& \left\|\left(A_{0}^{*} A_{0}+\beta B^{s}\right)^{-1} B^{s} h\right\| \\
& =\left\|B^{-s / 2}\left(A_{s}^{*} A_{s}+\beta I\right)^{-1} B^{s / 2} h\right\| \\
& \leq \frac{1}{f(s /(s+b))} \\
& \times\left\|\left(A_{s}^{*} A_{s}\right)^{s / 2(s+b)}\left(A_{s}^{*} A_{s}+\beta I\right)^{-1} B^{s / 2} h\right\| \\
& \leq \frac{1}{f(s /(s+b))}
\end{aligned}
$$

$$
\begin{aligned}
& \times\left\|\left(A_{s}^{*} A_{s}+\beta I\right)^{-1}\right\|\left\|\left(A_{s}^{*} A_{s}\right)^{s / 2(s+b)} B^{s / 2} h\right\| \\
\leq & \frac{g(s /(s+b))}{f(s /(s+b))} \frac{1}{\beta}\left\|B^{s / 2} h\right\|_{-s} \\
\leq & \frac{g(s /(s+b))}{f(s /(s+b))} \frac{1}{\beta}\|h\| .
\end{aligned}
$$

This completes the proof of the lemma.

Theorem 4. Let $e_{n, \alpha, s}^{\delta}$ and $q$ be as in (14) and (17), respectively; let $x_{n, \alpha, s}^{\delta}$ be as defined in (6) with $\delta \in\left(0, \delta_{0}\right]$. Then under Assumption 1 and Lemma 3, the following estimates hold for all $n \geq 0$ :

(a) $\left\|x_{n+1, \alpha, s}^{\delta}-x_{n, \alpha, s}^{\delta}\right\| \leq q^{n} \gamma_{\rho}$;

(b) $x_{n, \alpha, s}^{\delta} \in B_{r}\left(x_{0}\right)$.

Proof. If $x_{n, \alpha, s}^{\delta} \in B_{r}\left(x_{0}\right)$, then by Assumption 1,

$$
\begin{aligned}
x_{n+1, \alpha, s}^{\delta}-x_{n, \alpha, s}^{\delta} & \\
= & x_{n, \alpha, s}^{\delta}-x_{n-1, \alpha, s}^{\delta}-\left(A_{0}^{*} A_{0}+\beta B^{s}\right)^{-1} \\
& \times\left[A_{0}^{*}\left(F\left(x_{n, \alpha, s}^{\delta}\right)-F\left(x_{n-1, \alpha, s}^{\delta}\right)\right)\right. \\
& \left.+\alpha B^{s}\left(x_{n, \alpha, s}^{\delta}-x_{n-1, \alpha, s}^{\delta}\right)\right] \\
= & \left(A_{0}^{*} A_{0}+\beta B^{s}\right)^{-1} \\
& \times\left[A_{0}^{*} A_{0}\left(x_{n, \alpha, s}^{\delta}-x_{n-1, \alpha, s}^{\delta}\right)\right. \\
& -A_{0}^{*}\left(F\left(x_{n, \alpha, s}^{\delta}\right)-F\left(x_{n-1, \alpha, s}^{\delta}\right)\right) \\
& \left.+(\beta-\alpha) B^{s}\left(x_{n, \alpha, s}^{\delta}-x_{n-1, \alpha, s}^{\delta}\right)\right] \\
= & \left(A_{0}^{*} A_{0}+\beta B^{s}\right)^{-1} A_{0}^{*} \\
& \times \int_{0}^{1}\left[A_{0}-F^{\prime}\left(x_{n, \alpha, s}^{\delta}+t\left(x_{n, \alpha, s}^{\delta}-x_{n-1, \alpha, s}^{\delta}\right)\right)\right] \\
& \times\left(x_{n, \alpha, s}^{\delta}-x_{n-1, \alpha, s}^{\delta}\right) d t \\
+ & \left(A_{0}^{*} A_{0}+\beta B^{s}\right)^{-1}(\beta-\alpha) B^{s}\left(x_{n, \alpha, s}^{\delta}-x_{n-1, \alpha, s}^{\delta}\right)
\end{aligned}
$$

where

$$
\begin{aligned}
& \Gamma_{1}:=\left(A_{0}^{*} A_{0}+\beta B^{s}\right)^{-1} A_{0}^{*} \\
& \times \int_{0}^{1}\left[A_{0}-F^{\prime}\left(x_{n, \alpha, s}^{\delta}+t\left(x_{n, \alpha, s}^{\delta}-x_{n-1, \alpha, s}^{\delta}\right)\right)\right] \\
& \quad \times\left(x_{n, \alpha, s}^{\delta}-x_{n-1, \alpha, s}^{\delta}\right) d t
\end{aligned}
$$


and $\Gamma_{2}:=\left(A_{0}^{*} A_{0}+\beta B^{s}\right)^{-1}(\beta-\alpha) B^{s}\left(x_{n, \alpha, s}^{\delta}-x_{n-1, \alpha, s}^{\delta}\right)$, and hence by Assumption 1 and Lemma 3(a), we have

$$
\begin{aligned}
\left\|\Gamma_{1}\right\|= & \|-\left(A_{0}^{*} A_{0}+\beta B^{s}\right)^{-1} A_{0}^{*} A_{0} \\
& \times \int_{0}^{1} \Phi\left(x_{n-1, \alpha, s}^{\delta}+t\left(x_{n, \alpha, s}^{\delta}-x_{n-1, \alpha, s}^{\delta}\right),\right. \\
& \left.x_{0}, x_{n, \alpha, s}^{\delta}-x_{n-1, \alpha, s}^{\delta}\right) d t \| \\
\leq & \psi_{2}(s) k_{0} r\left\|x_{n, \alpha, s}^{\delta}-x_{n-1, \alpha, s}^{\delta}\right\|
\end{aligned}
$$

and by Lemma 3(b),

$$
\left\|\Gamma_{2}\right\| \leq \frac{\beta-\alpha}{\beta} \psi_{2}(s)\left\|x_{n, \alpha, s}^{\delta}-x_{n-1, \alpha, s}^{\delta}\right\| .
$$

Hence, by (19), (21), and (22), we have

$$
\begin{aligned}
& \left\|x_{n+1, \alpha, s}^{\delta}-x_{n, \alpha, s}^{\delta}\right\| \\
& \quad \leq \psi_{2}(s)\left(k_{0} r+\frac{\beta-\alpha}{\beta}\right)\left\|x_{n, \alpha, s}^{\delta}-x_{n-1, \alpha, s}^{\delta}\right\| \\
& \quad=q\left\|x_{n, \alpha, s}^{\delta}-x_{n-1, \alpha, s}^{\delta}\right\| \\
& \quad \leq q^{n}\left\|x_{1, \alpha, s}^{\delta}-x_{0, \alpha, s}^{\delta}\right\|=q^{n} e_{1, \alpha, s}^{\delta} .
\end{aligned}
$$

Next we show that $e_{1, \alpha, s}^{\delta}<\gamma_{\rho}$, using Assumption 1 and Lemma 3. Observe that

$$
\begin{aligned}
e_{1, \alpha, s}^{\delta}= & \left\|x_{1, \alpha, s}^{\delta}-x_{0, \alpha, s}^{\delta}\right\| \\
= & \left\|\left(A_{0}^{*} A_{0}+\beta B^{s}\right)^{-1} A_{0}^{*}\left(F\left(x_{0, \alpha, s}^{\delta}\right)-y^{\delta}\right)\right\| \\
= & \left\|B^{-s / 2}\left(A_{s}^{*} A_{s}+\beta I\right)^{-1} A_{s}^{*}\left(F\left(x_{0}\right)-y^{\delta}\right)\right\| \\
= & \| B^{-s / 2}\left(A_{s}^{*} A_{s}+\beta I\right)^{-1} A_{s}^{*} \\
& \times\left[y^{\delta}-y+F(\widehat{x})\right. \\
& \left.\quad-F\left(x_{0}\right)-A_{0}\left(\widehat{x}-x_{0}\right)+A_{0}\left(\widehat{x}-x_{0}\right)\right] \| \\
& +\left\|B^{-s / 2}\left(A_{s}^{*} A_{s}+\beta I\right)^{-1} A_{s}^{*}\left(y^{\delta}-y\right)\right\| \\
& \quad \times \int_{0}^{1}\left(F_{s}^{\prime}\left(x_{0}+t\left(\widehat{x}-x_{0}\right)\right)-A_{0}\right) \\
& +\left\|B^{-s / 2}\left(A_{s}^{*} A_{s}+\beta I\right)^{-1} A_{s}^{*} A_{0}\left(\widehat{x}-x_{0}\right)\right\|
\end{aligned}
$$

$$
\begin{aligned}
\leq & \frac{1}{f(s /(s+b))} \beta^{-b / 2(s+b)} \delta+\frac{g(s /(s+b))}{f(s /(s+b))} \frac{k_{0} \rho^{2}}{2} \\
& +\frac{g(s /(s+b))}{f(s /(s+b))} \rho \\
\leq & \overline{\psi_{2}(s)} \beta^{-b / 2(s+b)} \delta+\psi_{2}(s)\left(\frac{k_{0} \rho^{2}}{2}+\rho\right) \\
:= & \gamma_{\rho}<r .
\end{aligned}
$$

Hence, (a) follows from (23) and (24).

To prove (b), note that $\left\|x_{1, \alpha, s}^{\delta}-x_{0, \alpha, s}^{\delta}\right\| \leq \gamma_{\rho}<r$. Suppose $x_{m, \alpha, s}^{\delta} \in B_{r}\left(x_{0}\right)$ for some $m$; then

$$
\begin{aligned}
& \left\|x_{m+1, \alpha, s}^{\delta}-x_{0}\right\| \\
& \quad \leq\left\|x_{m+1, \alpha}^{\delta}-x_{m, \alpha, s}^{\delta}\right\|+\cdots+\left\|x_{1, \alpha, s}^{h, \delta}-x_{0}\right\| \\
& \quad \leq\left(q^{m}+q^{(m-1)}+\cdots+1\right) e_{1, \alpha, s}^{\delta} \\
& \quad \leq \frac{1}{1-q} e_{1, \alpha, s}^{\delta} \\
& \quad \leq \frac{\gamma_{\rho}}{1-q} \\
& \quad<r .
\end{aligned}
$$

Thus, by induction $x_{n, \alpha, s}^{\delta} \in B_{r}\left(x_{0}\right)$ for all $n \geq 0$. This proves (b).

Next we go to the main result of this section.

Theorem 5. Let $x_{n, \alpha, s}^{\delta}$ be as in (6), $\delta \in\left(0, \delta_{0}\right]$, and assumptions of Theorem 4 hold. Then $\left(x_{n, \alpha, s}^{\delta}\right)$ is a Cauchy sequence in $B_{r}\left(x_{0}\right)$ and converges, say, to $x_{\alpha, s}^{\delta} \in \overline{B_{r}\left(x_{0}\right)}$. Further $A_{0}^{*}\left(F\left(x_{\alpha, s}^{\delta}\right)-y^{\delta}\right)+$ $\alpha B^{s}\left(x_{\alpha, s}^{\delta}-x_{0}\right)=0$ and

$$
\left\|x_{n, \alpha, s}^{\delta}-x_{\alpha, s}^{\delta}\right\| \leq C q^{n}
$$

where $C=\gamma_{\rho} /(1-q)$.

Proof. Using relation (a) of Theorem 4, we obtain

$$
\begin{aligned}
& \left\|x_{n+m, \alpha, s}^{\delta}-x_{n, \alpha, s}^{\delta}\right\| \\
& \leq \sum_{i=0}^{i=m-1}\left\|x_{n+i+1, \alpha, s}^{\delta}-x_{n+i, \alpha, s}^{\delta}\right\| \\
& \leq \sum_{i=0}^{i=m-1} q^{(n+i)} e_{1, \alpha, s}^{\delta}
\end{aligned}
$$




$$
\begin{aligned}
= & q^{n} e_{1, \alpha, s}^{\delta}+q^{(n+1)} e_{1, \alpha, s}^{\delta} \\
& +\cdots+q^{(n+m)} e_{1, \alpha, s}^{\delta} \\
\leq & q^{n}\left(1+q+q^{2}+\cdots+q^{m}\right) e_{1, \alpha, s}^{\delta} \\
\leq & q^{n}\left(\frac{1}{1-q}\right) \gamma_{\rho} \\
\leq & C q^{n} .
\end{aligned}
$$

Thus, $x_{n, \alpha, s}^{\delta}$ is a Cauchy sequence in $B_{r}\left(x_{0}\right)$ and hence it converges, say, to $x_{\alpha, s}^{\delta} \in \overline{B_{r}\left(x_{0}\right)}$.

Now letting $n \rightarrow \infty$ in (6), we obtain

$$
A_{0}^{*}\left(F\left(x_{\alpha, s}^{\delta}\right)-y^{\delta}\right)+\alpha B^{s}\left(x_{\alpha, s}^{\delta}-x_{0}\right)=0 .
$$

This completes the proof.

The following assumption on source function and source condition is required to obtain the error estimates.

Assumption 6. There exists a continuous, strictly monotonically increasing function $\varphi:\left(0,\left\|A_{s}\right\|^{2}\right] \rightarrow(0, \infty)$ such that the following conditions hold:

(i) $\lim _{\lambda \rightarrow 0} \varphi(\lambda)=0$,

(ii) $\sup _{\lambda>0}(\alpha \varphi(\lambda) /(\lambda+\alpha)) \leq \varphi(\alpha)$ for all $\lambda \in\left(0,\left\|A_{s}\right\|^{2}\right]$, and

(iii) there exists $w \in X$ with $\|w\| \leq E_{2}$, such that

$$
\left(A_{s}^{*} A_{s}\right)^{s / 2(s+b)} B^{s / 2}\left(x_{0}-\widehat{x}\right)=\varphi\left(A_{s}^{*} A_{s}\right) w .
$$

Remark 7. If $x_{0}-\widehat{x} \in X_{t}$, for example, $\left\|x_{0}-\widehat{x}\right\|_{t} \leq E_{1}$, for some positive constant $E_{1}$ and $0 \leq t \leq 2 s+b$, then, we have $\left(A_{s}^{*} A_{s}\right)^{s / 2(s+b)} B^{s / 2}\left(x_{0}-\widehat{x}\right)=\varphi\left(A_{s}^{*} A_{s}\right) w$, where $\varphi(\lambda)=\lambda^{t /(s+b)}$, $w=\left(A_{s}^{*} A_{s}\right)^{(s-t) / 2(s+b)} B^{s / 2}\left(\widehat{x}-x_{0}\right)$, and $\|w\| \leq g((s-t) /(s+$ b)) $E_{1}:=E_{2}$.

Theorem 8. Let $x_{\alpha, s}^{\delta}$ be the solution of (7) and suppose Assumptions 1 and 6 hold; then

$$
\left\|x_{\alpha, s}^{\delta}-\hat{x}\right\| \leq \frac{\overline{\psi_{2}(s)}\left(\varphi(\alpha)+\alpha^{-b / 2(s+b)} \delta\right)}{1-\psi_{2}(s) k_{0} r} .
$$

Proof. Let $M=\int_{0}^{1} F^{\prime}\left(\widehat{x}+t\left(x_{\alpha, s}^{\delta}-\widehat{x}\right)\right) d t$. Then

$$
F\left(x_{\alpha, s}^{\delta}\right)-F(\widehat{x})=M\left(x_{\alpha, s}^{\delta}-\widehat{x}\right)
$$

Since $A_{0}^{*}\left(F\left(x_{\alpha, s}^{\delta}\right)-y^{\delta}\right)+\alpha B^{s}\left(x_{\alpha, s}^{\delta}-x_{0}\right)=0$, one can see that

$$
\begin{aligned}
\left(A_{0}^{*} A_{0}+\right. & \left.\alpha B^{s}\right)\left(x_{\alpha, s}^{\delta}-\widehat{x}\right) \\
= & \left(A_{0}^{*} A_{0}+\alpha B^{s}\right)\left(x_{\alpha, s}^{\delta}-\widehat{x}\right) \\
& -A_{0}^{*}\left(F\left(x_{\alpha, s}^{\delta}\right)-y^{\delta}\right)-\alpha B^{s}\left(x_{\alpha, s}^{\delta}-x_{0}\right) \\
= & A_{0}^{*}\left[A_{0}-M\right]\left(x_{\alpha, s}^{\delta}-\widehat{x}\right)+A_{0}^{*}\left(y^{\delta}-y\right) \\
& +\alpha B^{s}\left(x_{0}-\widehat{x}\right), \\
x_{\alpha, s}^{\delta}-\widehat{x}= & \left(A_{0}^{*} A_{0}+\alpha B^{s}\right)^{-1} \\
\times & {\left[A_{0}^{*}\left(A_{0}-M\right)\left(x_{\alpha, s}^{\delta}-\widehat{x}\right)\right.} \\
& \left.+A_{0}^{*}\left(y^{\delta}-y\right)+\alpha B^{s}\left(x_{0}-\widehat{x}\right)\right] \\
= & s_{1}+s_{2}+s_{3},
\end{aligned}
$$

where

$$
\begin{gathered}
s_{1}:=\left(A_{0}^{*} A_{0}+\alpha B^{s}\right)^{-1} A_{0}^{*}\left(A_{0}-M\right)\left(x_{\alpha, s}^{\delta}-\widehat{x}\right), \\
s_{2}:=\left(A_{0}^{*} A_{0}+\alpha B^{s}\right)^{-1} A_{0}^{*}\left(y^{\delta}-y\right), \\
s_{3}:=\left(A_{0}^{*} A_{0}+\alpha B^{s}\right)^{-1} \alpha B^{s}\left(x_{0}-\widehat{x}\right) .
\end{gathered}
$$

Note that by Assumption 1 and Lemma 3,

$$
\left\|s_{1}\right\| \leq \psi_{2}(s) k_{0} r\left\|x_{\alpha, s}^{\delta}-\widehat{x}\right\|,
$$

by Proposition 2,

$$
\left\|s_{2}\right\| \leq \overline{\psi_{2}(s)} \alpha^{-b / 2(s+b)} \delta,
$$

and by Assumption 6,

$$
\left\|s_{3}\right\| \leq \overline{\psi_{2}(s)} \varphi(\alpha) .
$$

Hence, by (34)-(36) and (32), we have

$$
\left\|x_{\alpha, s}^{\delta}-\hat{x}\right\| \leq \frac{\overline{\psi_{2}(s)}\left(\varphi(\alpha)+\alpha^{-b / 2(s+b)} \delta\right)}{1-\psi_{2}(s) k_{0} r} .
$$

This completes the proof of the theorem.

\subsection{Error Bounds under Source Conditions}

Theorem 9. Let $x_{n, \alpha, s}^{\delta}$ be as in (6). If assumptions in Theorems 5 and 8 hold, then

$$
\left\|\widehat{x}-x_{n, \alpha, s}^{\delta}\right\| \leq C q^{n}+\frac{\overline{\psi_{2}(s)}\left(\varphi(\alpha)+\alpha^{-b / 2(s+b)} \delta\right)}{1-\psi_{2}(s) k_{0} r},
$$

where $C$ is as in Theorem 5. Further if $n_{\delta}:=\min \left\{n: q^{n} \leq\right.$ $\left.\alpha^{-b / 2(s+b)} \delta\right\}$, then

$$
\left\|\widehat{x}-x_{n, \alpha, s}^{\delta}\right\| \leq C_{s}\left(\varphi(\alpha)+\alpha^{-b / 2(s+b)} \delta\right),
$$

where $C_{s}=C+\left(\overline{\psi_{2}(s)} /\left(1-\psi_{2}(s) k_{0} r\right)\right)$. 

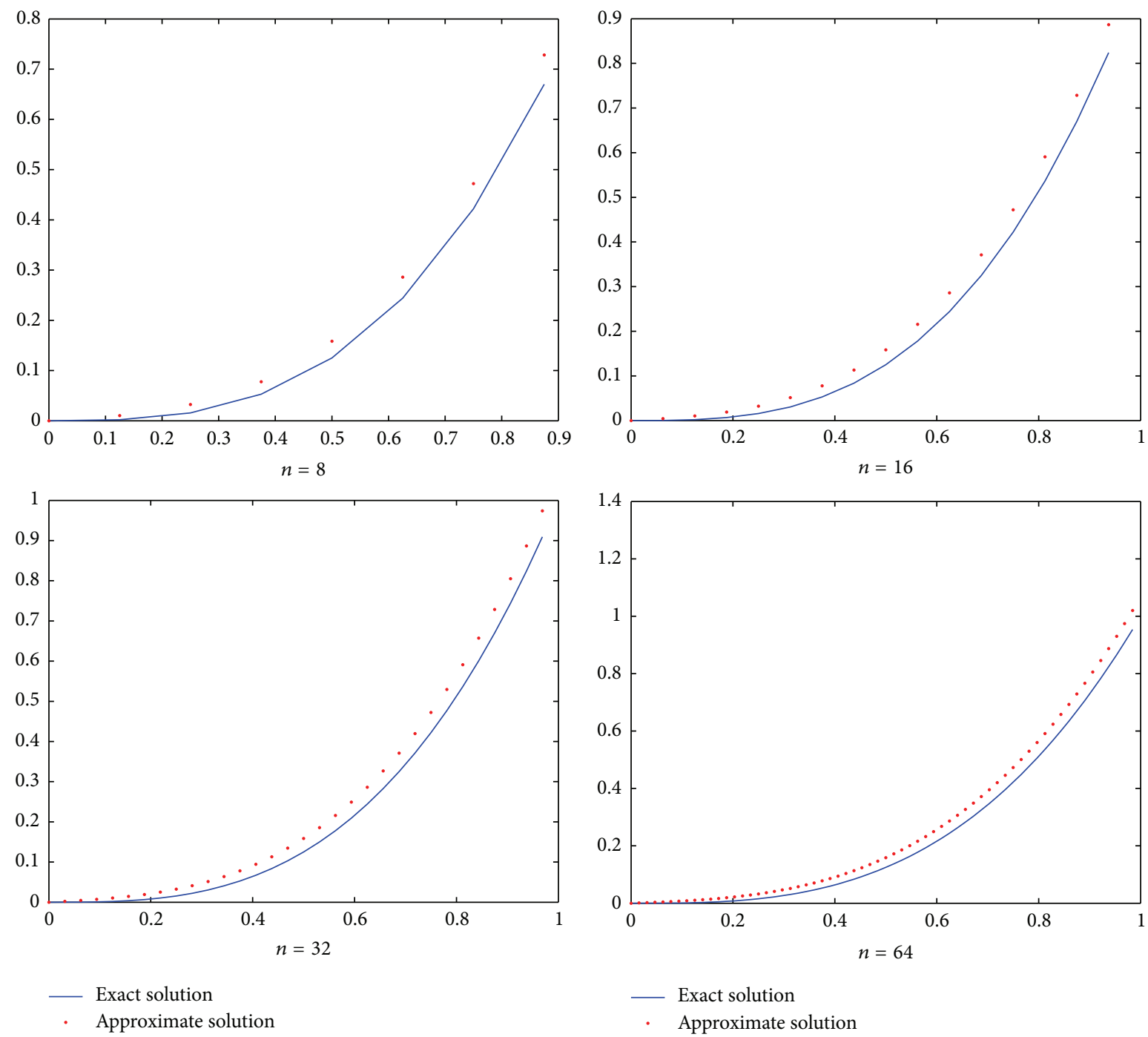

FIgURE 1: Curves of the exact and approximate solutions for $n=\{8,16,32,64\}$.

2.2. A Priori Choice of the Parameter. The error estimate $\varphi(\alpha)+\alpha^{-b / 2(s+b)} \delta$ in Theorem 9 attains minimum for the choice $\alpha:=\alpha(\delta, s, b)$ which satisfies $\varphi(\alpha)=\alpha^{-b / 2(s+b)} \delta$. Clearly $\alpha(\delta, s, b)=\varphi^{-1}\left(\psi_{s, b}^{-1}(\delta)\right)$, where

$$
\psi_{s, b}(\lambda)=\lambda\left[\varphi^{-1}(\lambda)\right]^{b / 2(s+b)}, \quad 0<\lambda \leq\left\|A_{s}\right\|^{2} .
$$

Thus, we have the following theorem.

Theorem 10. Suppose that all assumptions of Theorems 5 and 8 are fulfilled. For $\delta>0$, let $\alpha(\delta, s, b)=\varphi^{-1}\left(\psi_{s, b}^{-1}(\delta)\right)$, and let $n_{\delta}$ be as in Theorem 9. Then

$$
\left\|\widehat{x}-x_{n, \alpha, s}^{\delta}\right\| \leq O\left(\psi_{s, b}^{-1}(\delta)\right) .
$$

2.3. Adaptive Scheme and Stopping Rule. In this subsection, we consider the adaptive scheme suggested by Pereverzev and Schock in [17], modified suitably for choosing the parameter $\alpha$ which does not involve even the regularization method in an explicit manner.

Let $i \in\{0,1,2, \ldots, N\}$ and $\alpha_{i}=\mu^{i} \alpha_{0}$, where $\mu=\eta^{2(1+s / b)}$, $\eta>1$, and $\alpha_{0}=\delta^{2(1+s / b)}$. Let $n_{i}:=\min \left\{n: q^{n} \leq \alpha_{i}^{-b / 2(s+b)} \delta\right\}$, and let $x_{n_{i}, \alpha_{i}, s}^{\delta}$ be as defined in (6) with $\alpha=\alpha_{i}$ and $n=n_{i}$. Then, from Theorem 9, we have

$$
\left\|\widehat{x}-x_{n_{i}, \alpha_{i}, s}^{\delta}\right\| \leq C_{s}\left(\varphi\left(\alpha_{i}\right)+\alpha_{i}^{-b / 2(s+b)} \delta\right) .
$$

Further, let

$$
\begin{gathered}
l:=\max \left\{i: \varphi\left(\alpha_{i}\right) \leq \alpha_{i}^{-b / 2(s+b)} \delta\right\}<N, \\
k:=\max \left\{i:\left\|x_{n_{i}, \alpha_{i, s}}^{\delta}-x_{n_{j}, \alpha_{j, s}}^{\delta}\right\| \leq 4 C_{s} \alpha_{j}^{-b / 2(s+b)} \delta,\right. \\
j=0,1,2, \ldots, i-1\},
\end{gathered}
$$

where $C_{s}$ is as in Theorem 9. The proof of the following theorem is analogous to the proof of Theorem 4.4 in [31], so we omit the details. 

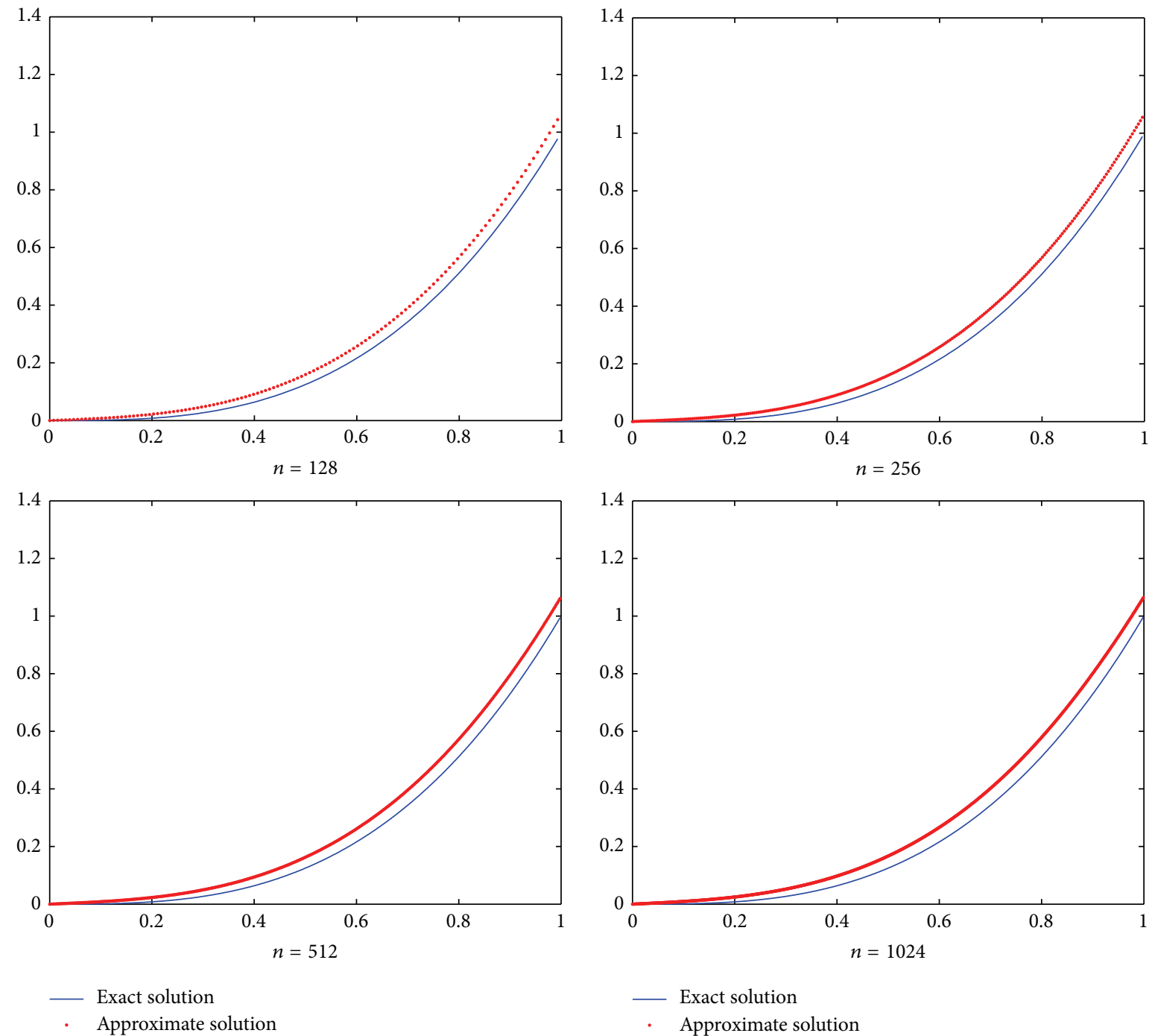

FIGURE 2: Curves of the exact and approximate solutions for $n=\{128,256,512,1024\}$.

TABLE 1: Iterations and corresponding error estimates with $\delta=1.8 E-5, \sigma=1$.

\begin{tabular}{llcccc}
\hline$n$ & $k$ & $n_{k}$ & $\alpha$ & $\left\|x_{n_{k}, \alpha, s}^{\delta}-\hat{x}\right\|$ & \multicolumn{1}{c}{$\frac{x_{n_{k}, \alpha, s}^{\delta}-\hat{x} \|}{(\delta)^{1 / 5}}$} \\
\hline 8 & 7 & 21 & 1.0606 & 0.9249 & 2.9244 \\
16 & 2 & 9 & 0.2857 & 1.0033 & 3.1724 \\
32 & 2 & 9 & 0.1431 & 1.0417 & 3.2940 \\
64 & 2 & 9 & 0.1429 & 1.0608 & 3.3543 \\
128 & 2 & 9 & 0.1428 & 1.0704 & 3.3847 \\
256 & 2 & 9 & 0.1428 & 1.0754 & 3.4005 \\
512 & 2 & 9 & 0.1428 & 1.0784 & 3.4172 \\
1024 & 2 & 9 & 0.1428 & 1.0807 & \\
\hline
\end{tabular}

Theorem 11. Let $x_{n, \alpha, s}^{\delta}$ be as in (6) with $\alpha=\alpha_{i}$ and $\delta \in\left(0, \delta_{0}\right]$, and assumptions in Theorem 9 hold. Let $l$ and $k$ be as defined in (43) and (44), respectively. Then $l \leq k$ and

$$
\left\|\widehat{x}-x_{n_{k}, \alpha_{k}, s}^{\delta}\right\| \leq 6 C_{s} \eta\left(\psi_{s, b}^{-1}(\delta)\right) .
$$

\section{Implementation of the Method}

Finally, the balancing algorithm associated with the choice of the parameter specified in Theorem 11 involves the following steps: 
TABLE 2: Iterations and corresponding error estimates with $\delta=1.8 E-5, \sigma=0.1$.

\begin{tabular}{llcccc}
\hline$n$ & $k$ & $n_{k}$ & $\alpha$ & $\left\|x_{n_{k}, \alpha, s}^{\delta}-\hat{x}\right\|$ & \multicolumn{1}{c}{$\frac{x_{n_{k}, \alpha, s}^{\delta}-\hat{x} \|}{(\delta)^{1 / 5}}$} \\
\hline 8 & 7 & 21 & 1.0605 & 0.9249 & 2.9246 \\
16 & 2 & 9 & 0.2856 & 1.0033 & 3.1726 \\
32 & 2 & 9 & 0.1431 & 1.0417 & 3.2942 \\
64 & 2 & 9 & 0.1429 & 1.0608 & 3.3546 \\
128 & 2 & 9 & 0.1428 & 1.0704 & 3.3850 \\
256 & 2 & 9 & 0.1428 & 1.0754 & 3.4008 \\
512 & 2 & 9 & 0.1428 & 1.0784 & 3.4101 \\
1024 & 2 & 9 & 0.1428 & 1.0807 & 3.4175 \\
\hline
\end{tabular}

(i) choose $\alpha_{0}>0$ such that $\delta_{0}<\left(\beta^{b / 2(s+b)} / 2 k_{0} \overline{\psi_{2}(s)}\right)$ $\left(\psi_{2}(s)+\left(\alpha_{0}^{2} / 2 \beta^{2}\right)\right)$ and $\eta>1$

(ii) choose $N$ big enough but not too large and $\alpha_{i}:=\mu^{i} \alpha_{0}$, $i=0,1,2, \ldots, N$, where $\mu=\eta^{2(1+s / a)} ;$

(iii) choose $\rho<\left(-1 / k_{0}\right)+\left(1 / k_{0} \psi_{2}(s)\right)$

$$
\sqrt{\psi_{2}(s)\left[\left(\left(\alpha^{2} / 2 \beta^{2}\right)+\psi_{2}(s)\right)-2 k_{0} \overline{\psi_{2}(s)} \beta^{-b / 2(s+b)} \delta\right]} \text {. }
$$

Algorithm 1.

(1) set $i=0$;

(2) choose $n_{i}=\min \left\{n: q^{n} \leq \alpha_{i}^{-a / 2(s+a)} \delta\right\}$;

(3) solve $x_{n_{i}, \alpha_{i}, s}^{\delta}$ by using the iteration (6);

(4) if $\left\|x_{n_{i}, \alpha_{i, s}}^{\delta}-x_{n_{j}, \alpha_{j, s}}^{\delta}\right\|>4 C_{s} \alpha_{j}^{-b / 2(s+b)} \delta, j<i$, then take $k=i-1$;

(5) else set $i=i+1$ and return to Step (2).

\section{Numerical Example}

Example 1. In this example, we consider a nonlinear integral operator, $F: D(F) \subset L^{2}(0,1) \rightarrow L^{2}(0,1)$, defined by

$$
F(x)(t):=\int_{0}^{1} k(t, s) x(s)^{3} d s=f(t)
$$

with

$$
k(t, s)= \begin{cases}(1-t) s, & 0 \leq s \leq t \leq 1 \\ (1-s) t, & 0 \leq t \leq s \leq 1\end{cases}
$$

The Fréchet derivative of $F$ is given by

$$
F^{\prime}(u) w=3 \int_{0}^{1} k(t, s)(u(s))^{2} w(s) d s
$$

In our computation, we take $y(t)=\left(t-t^{11}\right) / 110$ and $y^{\delta}=$ $y+\sigma(\|y\| /\|e\|) e$, where $e=\left(e_{i}\right)$ is a random vector with $e_{i} \sim$ $\aleph(0,1)$ and $\sigma>0$ is a constant [26]. Then the exact solution

$$
\widehat{x}(t)=t^{3} \text {. }
$$

We take $L: D \subset L^{2}(0,1) \rightarrow L^{2}(0,1)$ as

$$
\begin{gathered}
L x=\sum_{k=1}^{\infty} k\left\langle x, e_{k}\right\rangle e_{k} \quad \text { with } e_{k}(t)=\sqrt{2} \sin (k \pi t), \\
x_{0}(t)=t^{3}+\frac{t}{15}
\end{gathered}
$$

as our initial guess, so that the function $x_{0}-\widehat{x}$ satisfies the source condition $\left\|x_{0}-\widehat{x}\right\|_{t} \leq E, t \in[0,1 / 2)$ (see [20, Proposition 5.3]). Thus, we expect to have an accuracy of order at least $O\left(\delta^{1 / 5}\right)$.

As in [26], we use the $(n, n)$ matrix

$$
B:=B_{2}^{1 / 2} \quad \text { with } B_{2}=\frac{(n+1)^{2}}{\pi^{2}}\left(\begin{array}{cccc}
2 & -1 & & \\
-1 & \ddots & \ddots & \\
& \ddots & \ddots & -1 \\
& & -1 & 2
\end{array}\right)
$$

as a discrete approximation of the first-order differential operator (50).

We choose $\alpha_{0}=0.0171, \beta=1.19, k_{0}=1, s=2$, and $q=0.9$. The results of the computation are presented in Tables 1 and 2. The plots of the exact and the approximate solution obtained with $\delta=1.8 E-5$ are given in Figures 1 and 2 .

The last column of Tables 1 and 2 shows that the error $\left\|x_{\alpha_{k}, s}^{\delta}-\widehat{x}\right\|$ is of $O\left(\delta^{1 / 5}\right)$.

\section{Conclusion}

In this paper, we present an iterative regularization method for obtaining an approximate solution of a nonlinear ill-posed operator equation $F(x)=y$ in the Hilbert scale setting. Here $F: D(F) \subset X \rightarrow Y$ is a nonlinear operator and we assume that the available data is $y^{\delta}$ in place of exact data $y$. The convergence analysis was based on the center-type Lipschitz condition. We considered a Hilbert scale, $\left(X_{t}\right)_{t \in \mathbb{R}}$, generated by $B$, where $B: D(B) \subset X \rightarrow X$ is a linear, unbounded, self-adjoint, densely defined, and strictly positive operator on $X$. For choosing the regularization parameter $\alpha$, the adaptive scheme considered by Pereverzev and Schock in [17] was used. Finally, a numerical example is presented in support of our method which is found to be efficient. 


\section{Conflict of Interests}

The authors declare that there is no conflict of interests regarding the publication of this paper.

\section{Acknowledgment}

Ms. Monnanda Erappa Shobha thanks NBHM, DAE, Government of India, for the financial support.

\section{References}

[1] I. K. Argyros and S. Hilout, "A convergence analysis for directional two-step Newton methods," Numerical Algorithms, vol. 55, no. 4, pp. 503-528, 2010.

[2] I. K. Argyros and S. Hilout, "Weaker conditions for the convergence of Newton's method," Journal of Complexity, vol. 28, no. 3, pp. 364-387, 2012.

[3] I. K. Argyros, Y. J. Cho, and S. Hilout, Numerical Methods for Equations and its Applications, CRC Press, Taylor and Francis, New York, NY, USA, 2012.

[4] A. B. Bakushinsky and M. Y. Kokurin, Iterative Methods for Approximate Solution of Inverse Problems, Springer, Dordrecht, The Netherlands, 2004.

[5] H. W. Engl, K. Kunisch, and A. Neubauer, Regularization of Inverse Problems, Kluwer Academic Publishers, Dordrecht, The Netherlands, 1996.

[6] H. W. Engl, "Regularization methods for the stable solution of inverse problems," Surveys on Mathematics for Industry, vol. 3, no. 2, pp. 71-143, 1993.

[7] H. W. Engl, K. Kunisch, and A. Neubauer, "Convergence rates for Tikhonov regularisation of nonlinear ill-posed problems," Inverse Problems, vol. 5, no. 4, pp. 523-540, 1989.

[8] S. George, "Newton-type iteration for Tikhonov regularization of nonlinear ill-posed problems," Journal of Mathematics, vol. 2013, Article ID 439316, 9 pages, 2013.

[9] M. Hanke, "A regularizing Levenberg-Marquardt scheme, with applications to inverse groundwater filtration problems," Inverse Problems, vol. 13, no. 1, pp. 79-95, 1997.

[10] B. Kaltenbacher, "A note on logarithmic convergence rates for nonlinear Tikhonov regularization," Journal of Inverse and IllPosed Problems, vol. 16, no. 1, pp. 79-88, 2008.

[11] B. Kaltenbacher, A. Neubauer, and O. Scherzer, Iterative Regularization Methods for Nonlinear Ill-Posed Porblems, de Gruyter, Berlin, Germany, 2008.

[12] C. T. Kelley, Iterative Methods for Linear and Nonlinear Equations, SIAM, Philadelphia, Pa, USA, 1995.

[13] Q. Jin, "On a regularized Levenberg-Marquardt method for solving nonlinear inverse problems," Numerische Mathematik, vol. 115, no. 2, pp. 229-259, 2010.

[14] U. Tautenhahn, "On the method of Lavrentiev regularization for nonlinear ill-posed problems," Inverse Problems, vol. 18, no. 1, pp. 191-207, 2002.

[15] V. Vasin, "Irregular nonlinear operator equations: Tikhonov's regularization and iterative approximation," Journal of Inverse and Ill-Posed Problems, vol. 21, no. 1, pp. 109-123, 2013.

[16] V. Vasin and S. George, "Expanding the applicability of Tikhonov's regularization and iterative approximation for illposed problems," Journal of Inverse and Ill-Posed Problems, 2013.
[17] S. Pereverzev and E. Schock, "On the adaptive selection of the parameter in regularization of ill-posed problems," SIAM Journal on Numerical Analysis, vol. 43, no. 5, pp. 2060-2076, 2005.

[18] H. Egger and A. Neubauer, "Preconditioning Landweber iteration in Hilbert scales," Numerische Mathematik, vol. 101, no. 4, pp. 643-662, 2005.

[19] Q. Jin, "Error estimates of some Newton-type methods for solving nonlinear inverse problems in Hilbert scales," Inverse Problems, vol. 16, no. 1, pp. 187-197, 2000.

[20] S. Lu, S. V. Pereverzev, Y. Shao, and U. Tautenhahn, "On the generalized discrepancy principle for Tikhonov regularization in Hilbert scales," Journal of Integral Equations and Applications, vol. 22, no. 3, pp. 483-517, 2010.

[21] P. Mahale and M. T. Nair, "A simplified generalized GaussNewton method for nonlinear ill-posed problems," Mathematics of Computation, vol. 78, no. 265, pp. 171-184, 2009.

[22] P. Mathe and U. Tautenhahn, "Error bounds for regularization methods in Hilbert scales by using operator monotonicity," Far East Journal of Mathematical Sciences, vol. 24, no. 1, pp. 1-21, 2007.

[23] F. Natterer, "Error bounds for Tikhonov regularization in Hilbert scales," Applicable Analysis, vol. 18, no. 1-2, pp. 29-37, 1984.

[24] A. Neubauer, "On Landweber iteration for nonlinear ill-posed problems in Hilbert scales," Numerische Mathematik, vol. 85, no. 2, pp. 309-328, 2000.

[25] Q. Jin and U. Tautenhahn, "Inexact Newton regularization methods in Hilbert scales," Numerische Mathematik, vol. 117, no. 3, pp. 555-579, 2011.

[26] Q. Jin and U. Tautenhahn, "Implicit iteration methods in Hilbert scales under general smoothness conditions," Inverse Problems, vol. 27, no. 4, Article ID 045012, 2011.

[27] S. George and M. T. Nair, "Error bounds and parameter choice strategies for simplified regularization in Hilbert scales," Integral Equations and Operator Theory, vol. 29, no. 2, pp. 231-242, 1997.

[28] U. Tautenhahn, "On a general regularization scheme for nonlinear ill-posed problems: II. Regularization in Hilbert scales," Inverse Problems, vol. 14, no. 6, pp. 1607-1616, 1998.

[29] U. Tautenhahn, "Error estimates for regularization methods in Hilbert scales," SIAM Journal on Numerical Analysis, vol. 33, no. 6, pp. 2120-2130, 1996.

[30] Q. Jin, "On a class of frozen regularized Gauss-Newton methods for nonlinear inverse problems," Mathematics of Computation, vol. 79, no. 272, pp. 2191-2211, 2010.

[31] S. George, "On convergence of regularized modified Newton's method for nonlinear ill-posed problems," Journal of Inverse and Ill-Posed Problems, vol. 18, no. 2, pp. 133-146, 2010. 


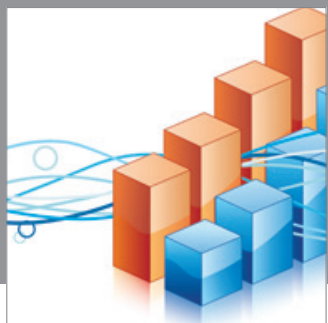

Advances in

Operations Research

mansans

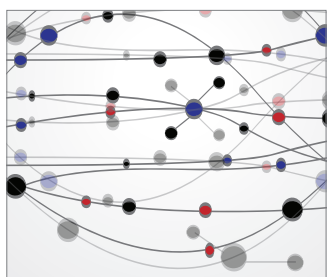

The Scientific World Journal
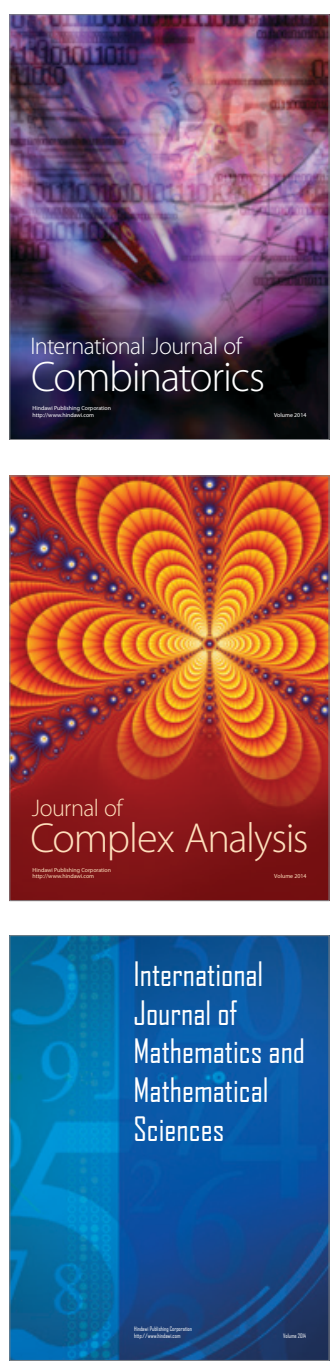
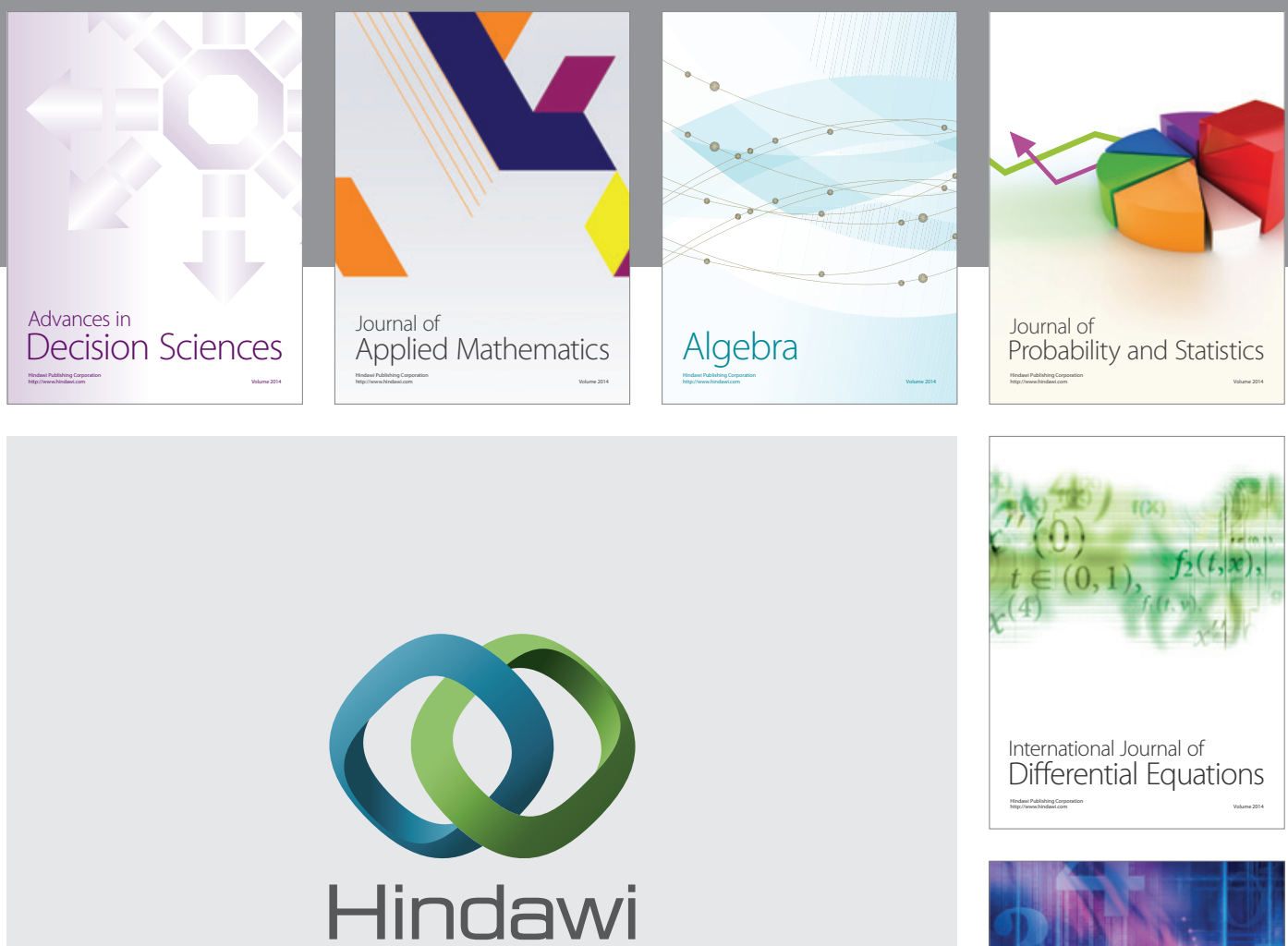

Submit your manuscripts at http://www.hindawi.com
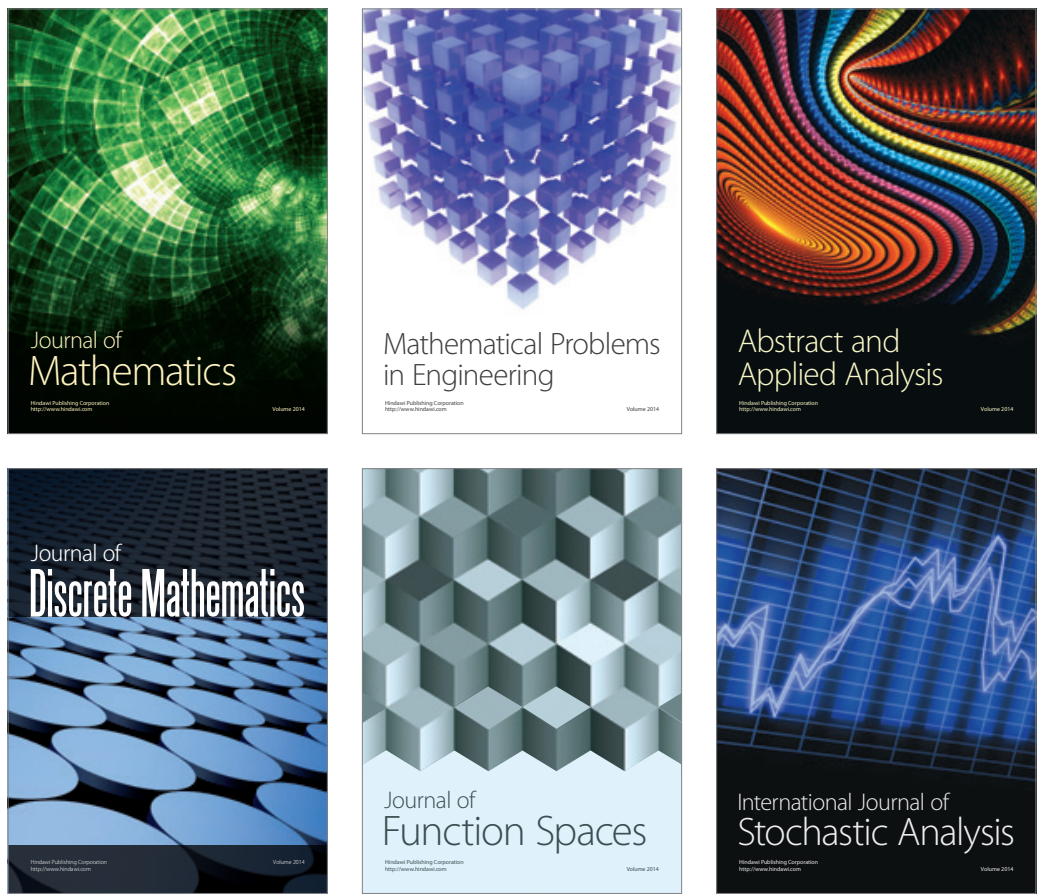

Journal of

Function Spaces

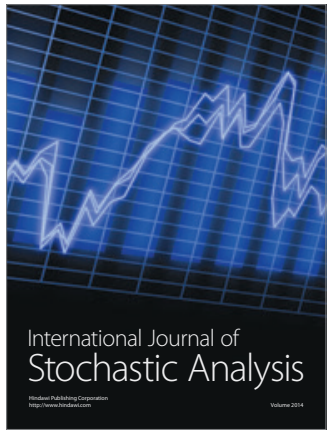

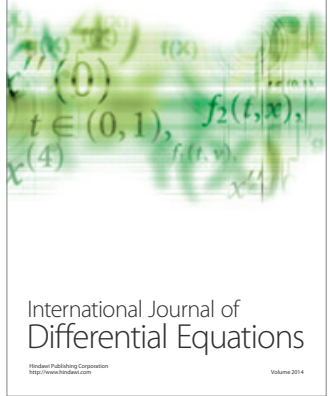
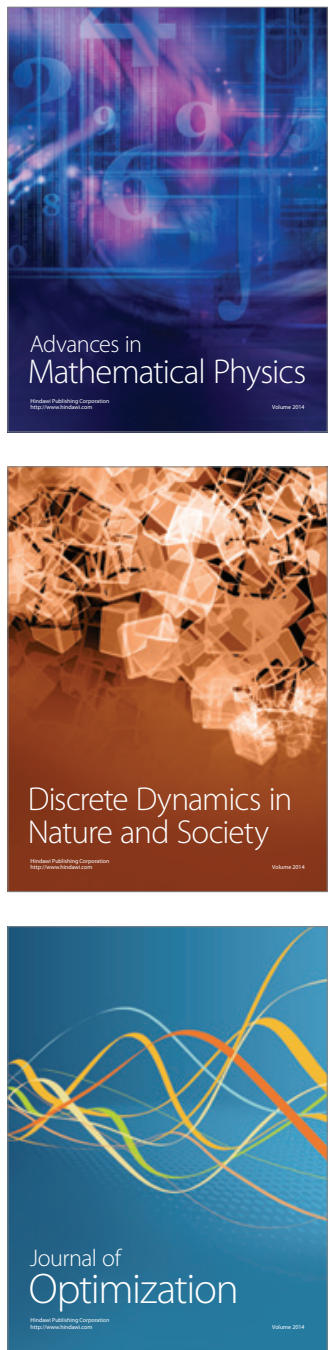